\title{
Modelling of natural convection in thin-disk lasers
}

\author{
Tom Dietrich ${ }^{1} \cdot$ Christoph Röcker $^{1} \cdot$ Thomas Graf $^{1} \cdot$ Marwan Abdou Ahmed $^{1}$
}

Received: 1 October 2019 / Accepted: 7 February 2020 / Published online: 22 February 2020

(c) The Author(s) 2020

\begin{abstract}
In this paper, we present a FEM-model that can be used to investigate the effects of thermally induced natural convection at the thin-disk laser crystal. Based on this simulation, we calculated the distribution of the refractive index of the ambient gas for the case of air and helium. By evaluating the optical path difference of a beam at normal incidence, the angular tilt (gas wedge) in the plane of the direction of convection as well as the spherical contribution (gas lens) was calculated for a set of different pump spot geometries and temperatures of the pumped area on the surface of the laser disk. Equations were derived that allow to simply calculate the tilt angle and the focal length of the gas lens for different temperatures of the disk and pump spot diameters for air as ambient medium.
\end{abstract}

\section{Introduction}

A major limitation for power scaling of solid-state lasers with good beam quality $\left(M^{2}<1.3\right)$ are thermally induced effects within and at the surface of the laser active medium. While the effects inside the crystal, e.g. thermal expansion and dispersion, are well understood [1,2], wavefront aberrations of the transmitted beam caused by thermally induced natural convection near the surface of the laser crystal so far have been investigated less extensively. This effect starts to play a significant role when it comes to laser oscillators and amplifiers with very high output powers (kW-class) and good beam quality. Even for thin-disk lasers, in which thermal effects are significantly reduced thanks to its efficient and longitudinal cooling concept [3], thermally induced convection can be a limiting factor that needs to be addressed [4].

A recent experimental investigation of the effect of the gas-lens introduced by convection in front of an Yb:YAG thin disk [5] showed that running the laser in $\mathrm{cw}$ and fundamental mode, the overall thermal lens can be reduced by about $33 \%$ when the system is operated in a vacuum chamber or in a Helium atmosphere rather than in air or Nitrogen atmosphere. In addition, the paper mentions numerical simulations that confirm the influence of the temperature of the

Christoph Röcker

christoph.roecker@ifsw.uni-stuttgart.de

1 Institut für Strahlwerkzeuge (IFSW), University of Stuttgart, Pfaffenwaldring 43, 70569 Stuttgart, Germany disk and the ambient gas on the resulting gas lens. Investigations that are more detailed are, however, required to understand the influence of both the surface temperature of the disk and the diameter of the heated area over a larger range. In fact, the knowledge of the influence of these parameters on the laser operation is of crucial importance for the design of advanced high-power fundamental-mode thin-disk oscillators [4] and amplifier systems [6,7]. Although a variety of different methods exists to estimate and compensate for thermally induced aberrations [4, 8-12], a complete elimination of aberrations introduced due to natural convection is only possible by operating the whole laser in vacuum [13], which, however, leads to an increased amount of complexity. If this effort is to be avoided, the influence of the convection needs to be considered in detail to be able to optimize the countermeasures.

In this paper, we, therefore, present an FEM-model that is used to investigate the effects of thermally induced natural convection at the thin-disk laser crystal. The FEM-model calculates a temperature distribution of the ambient gas resulting from the heat transfer at the heated boundaries and the transport by convection. From the resulting temperature distribution, one can extract the distribution of the refractive index in the ambient gas (e.g. air). By calculating the resulting optical path length of a beam incident on the heated laser crystal, we then extract the angular tilt which the beam suffers in the direction of convection (so-called 'gas wedge'effect) as well as the spherical contribution to the wave front distortions for different temperatures of the laser disk and different pump spot geometries. The quantitative knowledge 
on the angular and the spherical contribution to the wave front distortions is essential to be able to adapt the design of the laser cavity more precisely.

\section{Thermo-optical model}

To quantitatively determine the thermally induced linear ('gas wedge') and spherical ('gas lens') contributions to the wave front distortion of a transmitted laser beam, we have modelled the heated laser crystal and the surrounding atmosphere using the finite-element method (FEM) and the software COMSOL [14]. Figure 1 shows the geometric arrangement implemented in the FEM-model and the used coordinate system. The origin of the coordinate system is located at the center of the surface of the thin-disk laser crystal in contact with the ambient atmosphere. An Yb:YAG thin-disk crystal is modelled to be mounted on a diamond heat sink, surrounded by a gas filled volume measuring $40 \times 40 \times 40(\mathrm{~mm})^{3}$. The laser crystal was assumed to have a thickness of $110 \mu \mathrm{m}$ and a diameter of $15 \mathrm{~mm}$. The diamond heat sink was modeled to have a thickness of $2 \mathrm{~mm}$ and a diameter of $20 \mathrm{~mm}$. The cooling temperature of the backside of the diamond heat sink is set to $15^{\circ} \mathrm{C}$. The heat conductivity of the $\mathrm{Yb}$ :YAG crystal and the diamond heatsink was $8 \mathrm{~W} /(\mathrm{mK})$ [15] and $1800 \mathrm{~W} /(\mathrm{mK})$ [16]. A perfect thermal contact between disk material and heatsink is assumed in the model. The pumped region is modelled as a homogeneous area with an increased temperature at the uncooled surface of the crystal. The size of the heated, i.e. pumped, area and its temperature on the surface of the crystal can be varied in the simulation. The boundaries of the gas-filled volume are modelled as open boundaries. Other thermo-mechanical effects, e.g. thermal expansion of the laser crystal or the heat

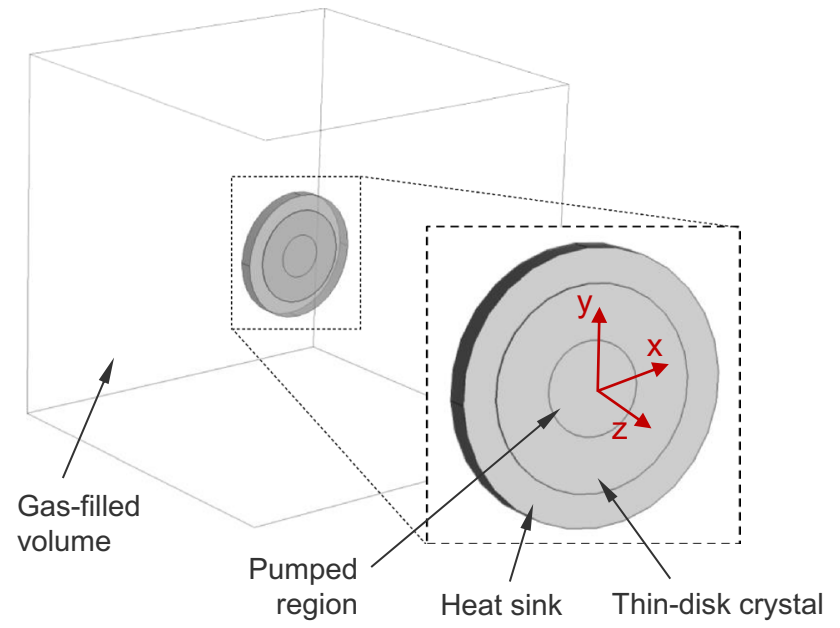

Fig. 1 Geometric arrangement of the thin-disk laser crystal and the surrounding atmosphere as modelled by the FEM sink, are not taken into account in this model. An influence of the surrounding mechanical geometry, i.e. pump light multipass arrangement, was also not taken into account in the model.

For the simulation of natural convection, COMSOL's heat transfer module (heat transfer equations) was coupled with its fluid dynamics module (Navier-Stokes-equations). The characteristic buoyancy force of the natural convection was modeled as a volume force acting on the gas volume. This volume force depends on the temperature-dependent mass density of the gas and can simply be calculated by $f=F / V=-\rho \cdot g$, with the gravitational acceleration $g=9.81 \mathrm{~m} /$ $\mathrm{s}^{2}$ and the density $\rho$ of the gas. The direction of the force was oriented to act in the $-y$-direction (as depicted in Fig. 1).

The calculated distribution of the temperature and the velocity field for air as ambient gas is shown in Fig. 2. For this simulation, the temperature of the uncooled surface of the pumped area of the disk was set to $140{ }^{\circ} \mathrm{C}$, which roughly corresponds to a pump power density of approx. $5 \mathrm{~kW} / \mathrm{cm}^{2}$ at a pump wavelength of $940 \mathrm{~nm}$ and approx. $7-8 \mathrm{~kW} / \mathrm{cm}^{2}$ at a pump wavelength of $969 \mathrm{~nm}$ (fluorescence operation). The diameter of the pumped area (pump spot diameter) was chosen to be $7 \mathrm{~mm}$. The initial temperature and pressure of the ambient air were set to $20^{\circ} \mathrm{C}$ and $1 \mathrm{~atm}$, respectively. Figure 2a shows the temperature distribution in the YZ-plane (parallel to the direction of convection) and Fig. $2 \mathrm{~b}$ shows the temperature distribution in the XZ-plane (perpendicular to the direction of convection). Figure 2c shows a cross-section of the velocity field of the heated air in the YZ-plane. The arrows indicate the direction of airflow, their color the temperature of the air. The arrow length qualitatively shows the velocity of the air.

The temperature dependence of the refractive index of air at a wavelength of $1030 \mathrm{~nm}$, at a pressure of $1 \mathrm{~atm}$, and a relative humidity of $20 \%$, was derived from [17] and approximated by

$n_{\text {Air }}=1+2.83707 \cdot 10^{-4}-7.88613 \cdot 10^{-7^{\circ}} \mathrm{C}^{-1} \cdot\left(T-T_{0}\right)$,

where $T$ is the temperature and $T_{0}=0{ }^{\circ} \mathrm{C}$. Figure 3 shows the calculated distribution of the refractive index of the ambient gas in the YZ- and the XZ-plane. A clearly asymmetric distribution of the refractive index can be observed in the YZ-plane, whereas the distribution of the refractive index is symmetrical in the XZ-plane. The corresponding distribution of the optical path length for a beam incident along the $Z$-axis (single pass, i.e. from left to right at different positions in $x$ - (Fig. 3a) and $y$-direction (Fig. 3b), respectively) were calculated from this. Subtracting the OPL of the same path but without pumping the disk (cold disk) leads the optical path difference (OPD), as shown in Fig. 4a, b.

The linear part of the wavefront aberration occurring in the YZ-plane shown in Fig. 4a was extracted by applying a 
(a)

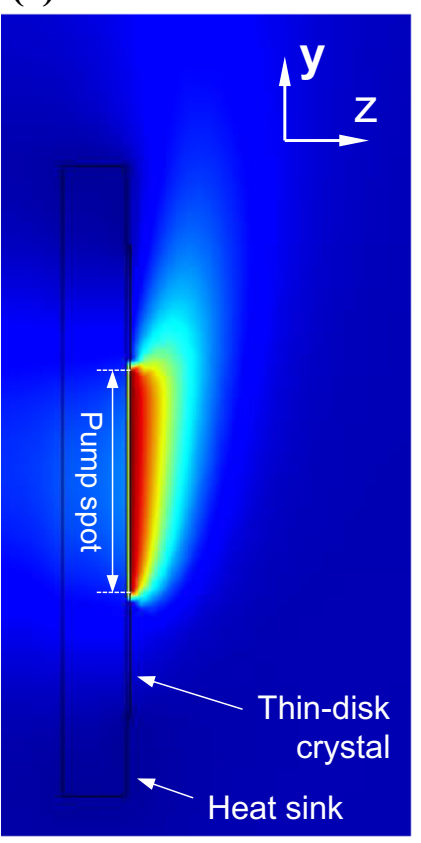

(b)

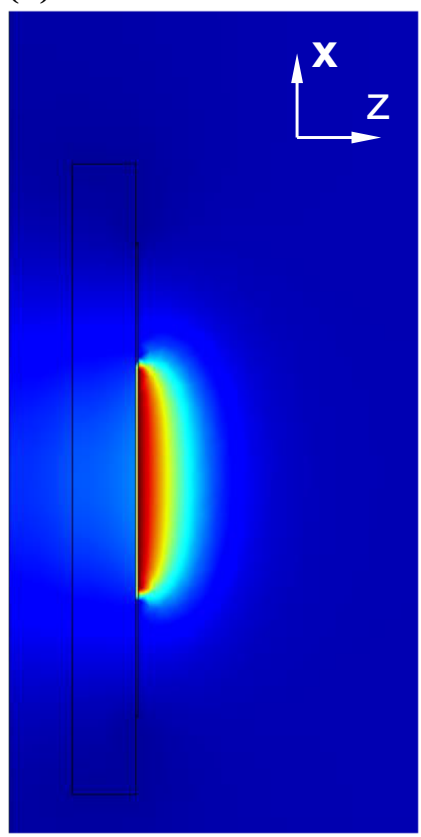

(c)

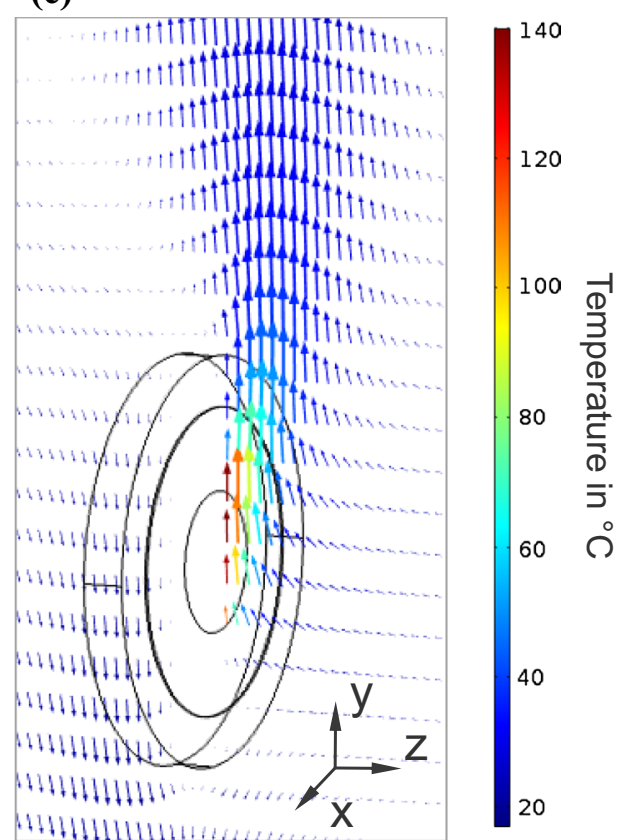

Fig. 2 Calculated distribution of the temperature of the ambient air, (a) in the YZ-plane (parallel to the direction of convection) and (b) in the XZ-plane (perpendicular to the direction of convection). Calculated velocity field of the ambient air in the YZ-plane (c)

(a)

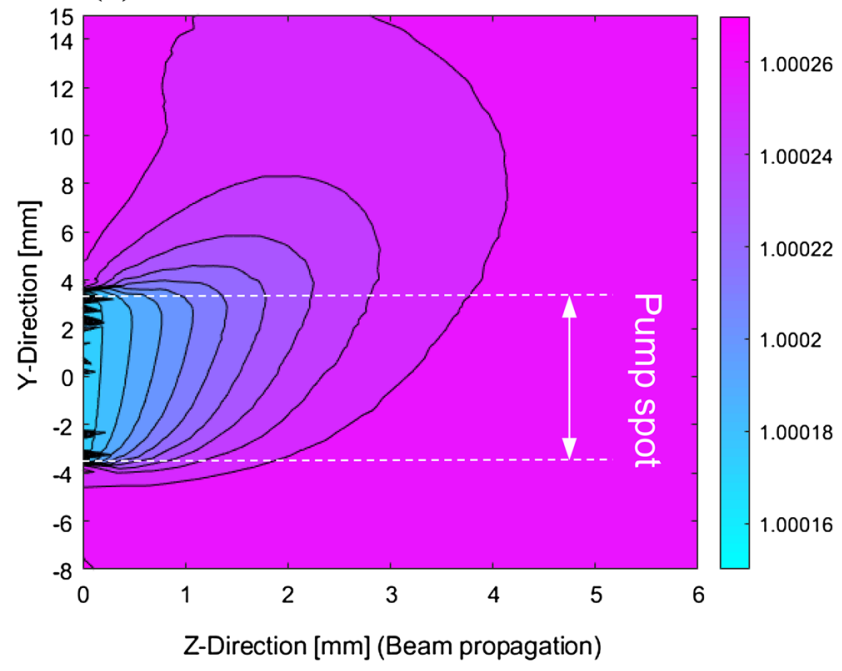

(b)

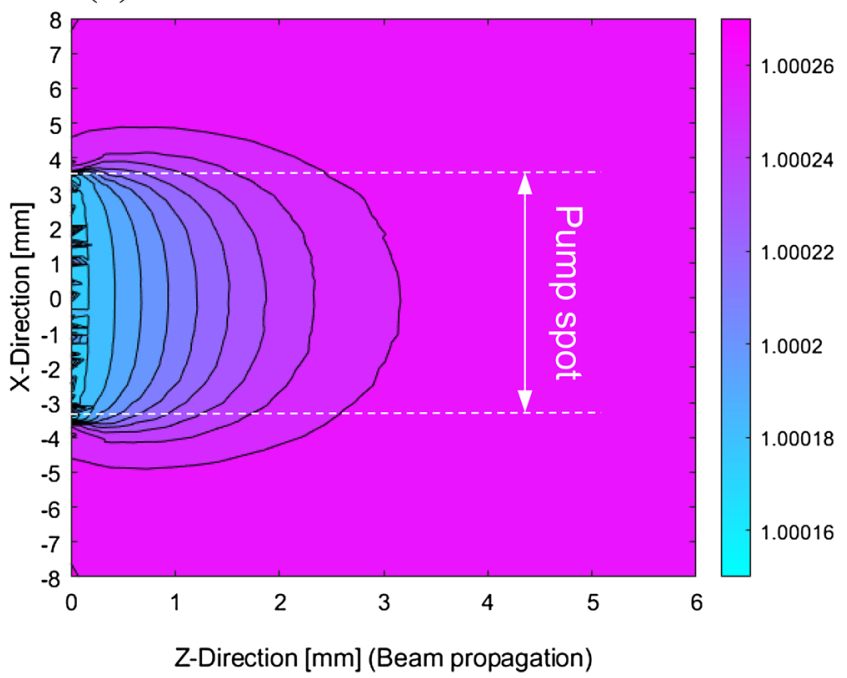

Fig. 3 Calculated distribution of the refractive index $n_{\text {Air }}$ (color coded) in the YZ-plane (a) and in the XZ-plane (b)

linear fit to the central area of the OPD, shown as dashed line. The edges of the fitted area are marked as red dotted lines. The angular tilt $\theta$ experienced by an incident wavefront is determined by the slope of the fitted curve. The OPD in the XZ-plane exhibits no linear contribution ('gas wedge'), shown in Fig. 4b. A quadratic fit to the central area of the $\operatorname{OPD}(\mathrm{x})$ reveals a spherical contribution ('gas lens'). Subtracting the linear contribution ('gas wedge') of the OPD(y) in the YZ-plane (Fig. 4a) leads to the residual OPD (y) shown in Fig. 4c, which exhibits a further spherical contribution. Note that in Fig. 3, close to the disk surface at $z=0$, black, triangle-shaped artifacts occur in the color map plot. These artifacts are generated during data transfer of the non-equidistant FEM-grid to an matrix-based format with equidistant spacing. They have no physical meaning and are not influencing the fit result. 
(a)

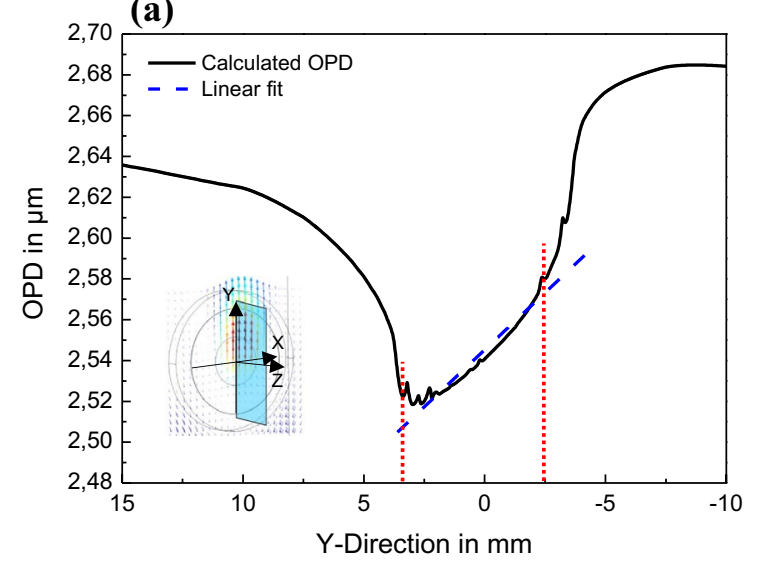

(b)

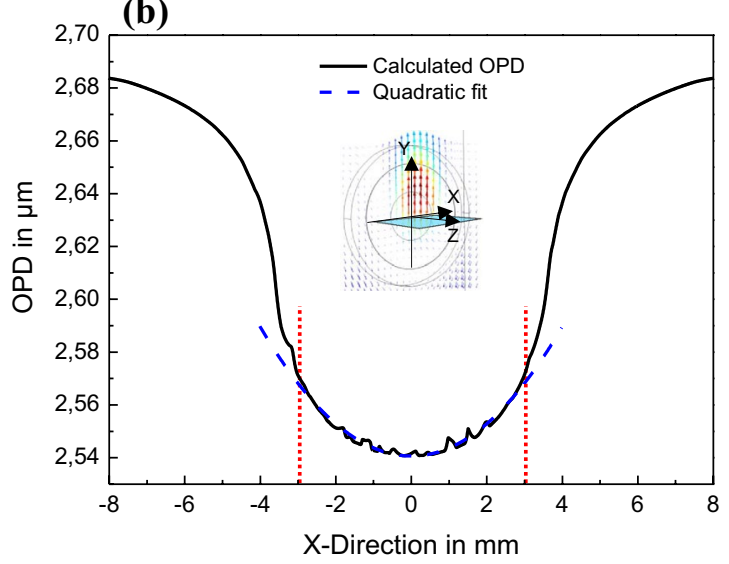

(c)

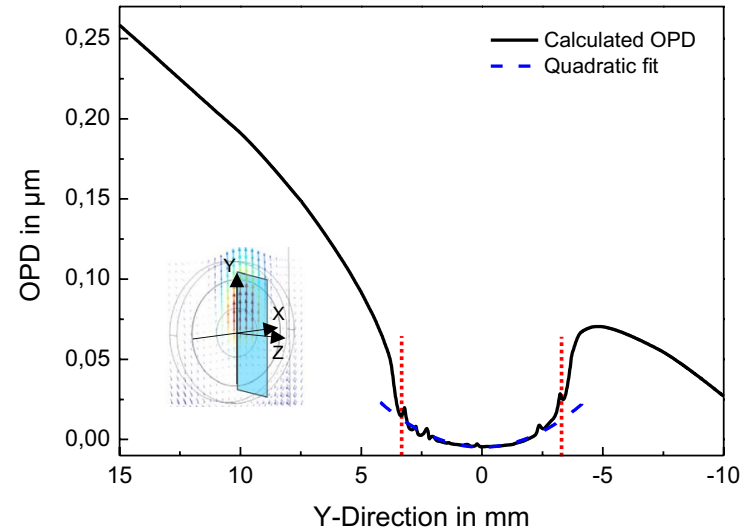

Fig. 4 Calculated OPD in the YZ-plane (a) and XZ-plane (b). The slope of the linear fit (dashed line) to the curve in a determines the tilt that is induced to the laser beam. In $\mathbf{b}$ a quadratic fit curve is shown in the plot (blue dashed line) by which the spherical contribution to the wavefront distortion was calculated. The residual OPD after sub-

To derive an empirical formula for the dependence of the air-induced tilt $\theta$ and the spherical distortions of the wavefront on the temperature of the crystal's surface and the diameter of the pump spot, simulations were performed for a number of different temperatures ranging from $T_{\text {Disk }}=40{ }^{\circ} \mathrm{C}$ to $160{ }^{\circ} \mathrm{C}$ and assuming diameters of the pump spot of $4 \mathrm{~mm}, 6 \mathrm{~mm}, 7 \mathrm{~mm}$ and $9 \mathrm{~mm}$.

From these simulation results one can empirically derive the relation

$$
\begin{aligned}
& \theta\left(T, d_{\mathrm{pump}}\right)=\left(A_{\mathrm{W}-\mathrm{YZ}, 1} \cdot d^{2}+B_{\mathrm{W}-\mathrm{YZ}, 1} \cdot d+C_{\mathrm{W}-\mathrm{YZ}, 1}\right) \cdot T\left[{ }^{\circ} \mathrm{C}\right] \\
& \quad+\left(A_{\mathrm{W}-\mathrm{YZ}, 2} \cdot d^{2}+B_{\mathrm{W}-\mathrm{YZ}, 2} \cdot d+C_{\mathrm{W}-\mathrm{YZ}, 2}\right),
\end{aligned}
$$

traction of the linear contribution in the YZ-plane (as shown in a) is shown in (c). A quadratic fit curve (blue dashed line) was applied to extract the residual spherical contribution to the distortion of the wavefront in the YZ-plane. The extend of the corresponding fit range is indicated by red dotted limits

where fit coefficients are given in Table 1.

Figure 5 shows the calculated air-induced tilt $\theta$ as a function of the temperature of the uncooled side of the thin-disk. The air-induced tilt $\theta$ is found to also strongly depend on the diameter of the pump spot, decreasing with increasing diameter of the pump spot. The dashed lines in Fig. 5 were calculated using Eq. 2. The use of this equation for the design of thin-disk lasers is more convenient than having to resort to numerical simulations.

The thermally induced focal length $f^{\prime}$ and the corresponding refractive power $\mathrm{D}$ of the gas lens that causes the spherical deformation of the wavefront are shown in Fig. 6 both for the YZ-plane (plane of convection, Fig. 6a, b) and the

Table 1 Fit coefficients of Eq. 2

\begin{tabular}{llllll}
\hline$A_{\mathrm{W}-\mathrm{YZ}, 1}$ & $2.74 \cdot \mathrm{E}-03$ & {$\left[\mu \mathrm{rad} /\left({ }^{\circ} \mathrm{C} \cdot \mathrm{mm}^{2}\right)\right]$} & $A_{\mathrm{W}-\mathrm{YZ}, 2}$ & $-2.36 \cdot \mathrm{E}-01$ & {$\left[\mu \mathrm{rad} / \mathrm{mm}^{2]}\right.$} \\
$B_{\mathrm{W}-\mathrm{YZ}, 1}$ & $-5.31 \cdot \mathrm{E}-02$ & {$\left[\mu \mathrm{rad} /\left({ }^{\circ} \mathrm{C} \cdot \mathrm{mm}\right)\right]$} & $B_{\mathrm{W}-\mathrm{YZ}, 2}$ & 3.77 & {$[\mu \mathrm{rad} / \mathrm{mm}]$} \\
$C_{\mathrm{W}-\mathrm{YZ}, 1}$ & $3.25 \cdot \mathrm{E}-01$ & {$\left[\mu \mathrm{rad} /{ }^{\circ} \mathrm{C}\right]$} & $C_{\mathrm{W}-\mathrm{YZ}, 2}$ & -15.19 & {$[\mu \mathrm{rad}]$} \\
\hline
\end{tabular}


Fig. 5 Calculated air-induced tilt $\theta$ over the temperature of the uncooled surface of the thindisk crystal for different pump spot diameters. The dashed lines correspond to Eq. 2 using the fit parameter given in Table 3
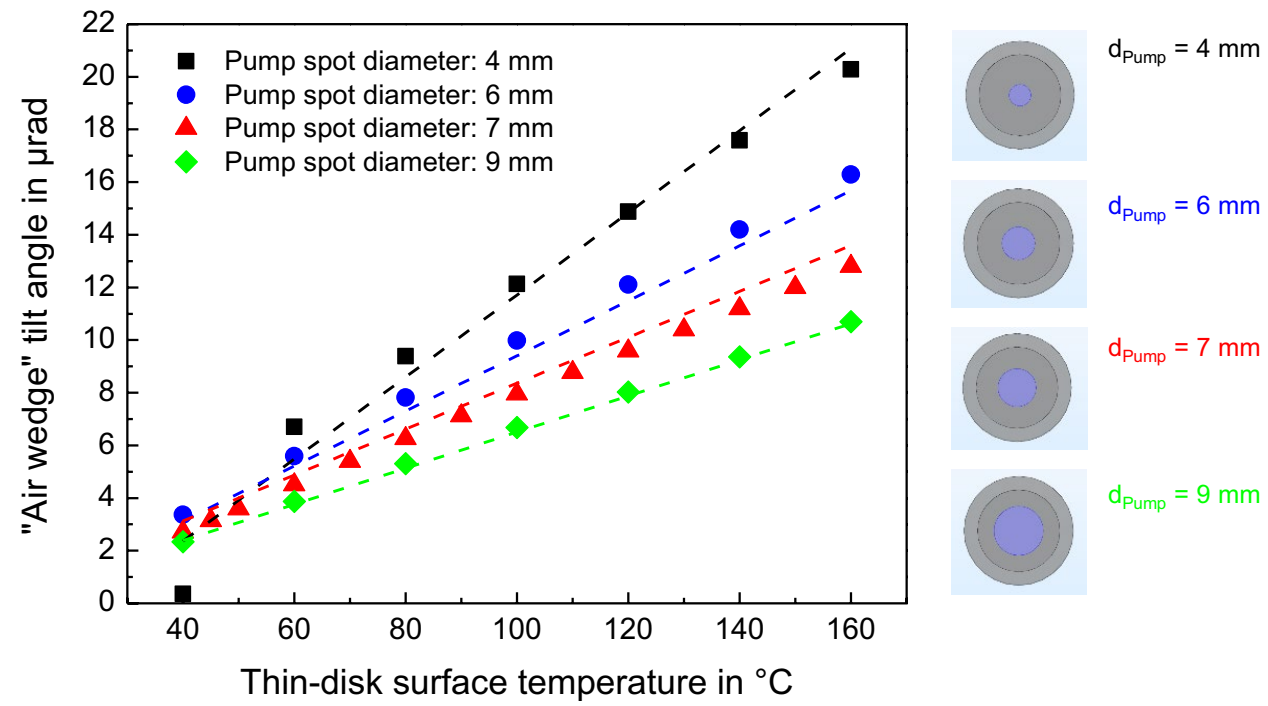

XZ-plane (Fig. 6c, d). It can be seen, that the dioptric power of the gas lens increases with increasing temperature of the surface of the laser crystal and with decreasing diameter of the pump spot. Additionally, the focal length $f^{\prime}$ (and the corresponding refractive power D) of the gas lens exhibits a significant difference in magnitude in the $\mathrm{YZ}$ and $\mathrm{XZ}$-plane.

Again the simulation results obtained for the YZ- and the XZ-plane can be fitted by the empirical equations (a)

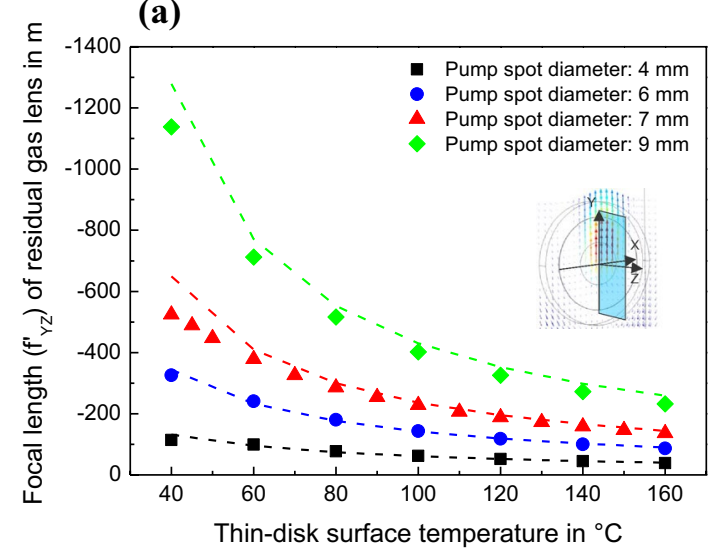

(c)

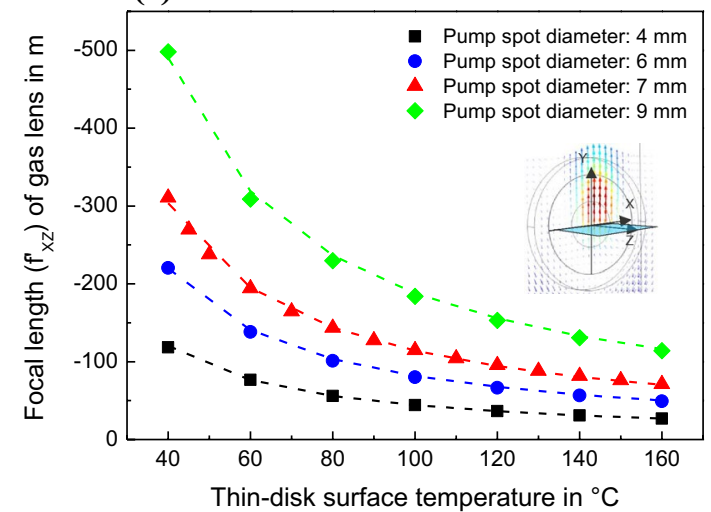

Fig. 6 Calculated focal length and refractive power of the gas lens in the YZ-plane (a) and (b), respectively, and calculated focal length and refractive power of the gas lens in the XZ-plane (c) and (d), (b)

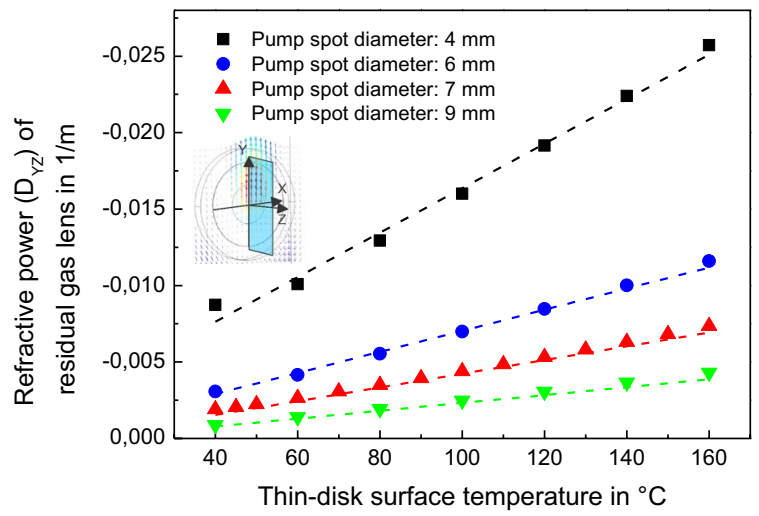

(d)

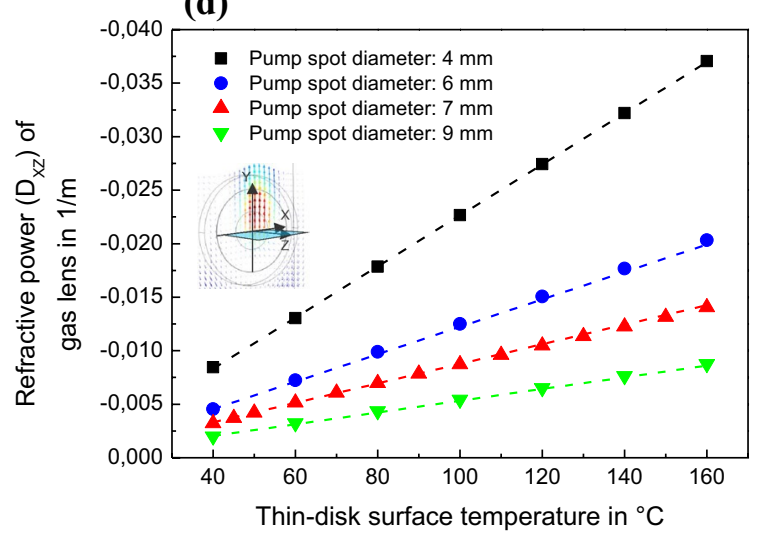

respectively. The dashed curves in (a) and (b) are given by Eq. 3 and the ones in (c) and (d) by Eq. 4 
Table 2 Fit coefficients of Eq. 3

\begin{tabular}{llllll}
\hline$A_{\mathrm{L}-\mathrm{YZ}, 1}$ & $-4.80 \cdot \mathrm{E}-6$ & {$\left[1 /\left(\mathrm{m} \cdot{ }^{\circ} \mathrm{C} \cdot \mathrm{mm}^{2}\right)\right]$} & $A_{\mathrm{L}-\mathrm{YZ}, 2}$ & $-1.38 \cdot \mathrm{E}-4$ & {$\left[1 /\left(\mathrm{m} \cdot \mathrm{mm}^{2}\right)\right]$} \\
$B_{\mathrm{L}-\mathrm{YZ}, 1}$ & $8.64 \cdot \mathrm{E}-5$ & {$\left[1 /\left(\mathrm{m} \cdot{ }^{\circ} \mathrm{C} \cdot \mathrm{mm}\right)\right]$} & $B_{\mathrm{L}-\mathrm{YZ}, 2}$ & $2.20 \cdot \mathrm{E}-3$ & {$[1 /(\mathrm{m} \cdot \mathrm{mm})]$} \\
$C_{\mathrm{L}-\mathrm{YZ}, 1}$ & $-4.15 \cdot \mathrm{E}-4$ & {$\left[1 /\left(\mathrm{m} \cdot{ }^{\circ} \mathrm{C}\right)\right]$} & $C_{\mathrm{L}-\mathrm{YZ}, 2}$ & $-8.40 \cdot \mathrm{E}-3$ & {$[1 / \mathrm{m}]$} \\
\hline
\end{tabular}

Table 3 Fit coefficients of Eq. 4

\begin{tabular}{llllll}
\hline$A_{\mathrm{L}-\mathrm{XZ}, 1}$ & $-6.17 \cdot \mathrm{E}-6$ & {$\left[1 /\left(\mathrm{m} \cdot{ }^{\circ} \mathrm{C} \cdot \mathrm{mm}^{2}\right)\right]$} & $\mathrm{A}_{\mathrm{L}-\mathrm{XZ}, 2}$ & $3.73 \cdot \mathrm{E}-5$ & {$\left[1 /\left(\mathrm{m} \cdot \mathrm{mm}^{2}\right)\right]$} \\
$B_{\mathrm{L}-\mathrm{XZ}, 1}$ & $1.17 \cdot \mathrm{E}-4$ & {$\left[1 /\left(\mathrm{m} \cdot{ }^{\circ} \mathrm{C} \cdot \mathrm{mm}\right)\right]$} & $\mathrm{B}_{\mathrm{L}-\mathrm{XZ}, 2}$ & $-7.00 \cdot \mathrm{E}-4$ & {$[1 /(\mathrm{m} \cdot \mathrm{mm})]$} \\
$C_{\mathrm{L}-\mathrm{XZ}, 1}$ & $-6.08 \cdot \mathrm{E}-04$ & {$\left[1 /\left(\mathrm{m} \cdot{ }^{\circ} \mathrm{C}\right)\right]$} & $\mathrm{C}_{\mathrm{L}-\mathrm{XZ}, 2}$ & $3.43 \cdot \mathrm{E}-3$ & {$[1 / \mathrm{m}]$} \\
\hline
\end{tabular}

$$
\begin{aligned}
1 / f_{\mathrm{YZ}}= & D_{\mathrm{YZ}}\left(T, d_{\text {pump }}\right) \\
= & \left(A_{\mathrm{L}-\mathrm{YZ}, 1} \cdot d^{2}+B_{\mathrm{L}-\mathrm{YZ}, 1} \cdot d+C_{\mathrm{L}-\mathrm{YZ}, 1}\right) \cdot T\left[{ }^{\circ} \mathrm{C}\right] \\
& +\left(A_{\mathrm{L}-\mathrm{YZ}, 2} \cdot d^{2}+B_{\mathrm{L}-\mathrm{YZ}, 2} \cdot d+C_{\mathrm{L}-\mathrm{YZ}, 2}\right),
\end{aligned}
$$

and

$$
\begin{aligned}
1 / f_{\mathrm{XZ}}= & D_{\mathrm{XZ}}\left(T, d_{\text {pump }}\right) \\
= & \left(A_{\mathrm{L}-\mathrm{XZ}, 1} \cdot d^{2}+B_{\mathrm{L}-\mathrm{XZ}, 1} \cdot d+C_{\mathrm{L}-\mathrm{XZ}, 1}\right) \cdot T\left[{ }^{\circ} \mathrm{C}\right] \\
& +\left(A_{\mathrm{L}-\mathrm{XZ}, 2} \cdot d^{2}+B_{\mathrm{L}-\mathrm{XZ}, 2} \cdot d+C_{\mathrm{L}-\mathrm{XZ}, 2}\right),
\end{aligned}
$$

where the fit parameters of Eqs. 3 and 4 are summarized in Tables 2 and 3 , respectively.

The quantitative knowledge on the angular and the spherical contribution to the wave-front distortions can be used to adapt the design of the laser cavity at an early stage of the design process, e.g. by implementing additional optical components which compensate for the presented effects.

To validate our results experimentally, a sophisticated setup has been developed and promising first results have been generated. Although a good agreement of simulated and experimental results was found, a clear distinction of the influence of mechanical and optical effects is currently part of ongoing investigations.

Additionally, it is important to note that a reduction of the air pressure significantly reduces the absolute value of $n_{\text {Air }}$ as well as $\mathrm{d} n_{\text {Air }} / \mathrm{d} T$ and consequently decreases the tilt induced to the laser beam as well as the dioptric power of the thermally induced spherical contribution to the wavefront distortion. The influence of the humidity on the refractive index of air (at a temperature of $20{ }^{\circ} \mathrm{C}$ and a pressure of $1 \mathrm{~atm})$ was found to be minor.

Finally, we have performed similar considerations also for thin-disk lasers operated in a helium atmosphere using the information published in [18] to calculate the refractive index. Both the tilt induced to the laser beam as well as the dioptric power of the thermally induced spherical contribution to the wavefront distortion were found to be about one order of magnitude smaller than the values obtained in ambient air. These findings confirm the results presented in [5].

\section{Conclusion}

In conclusion, we have presented numerical FEM investigations and therefrom derived empirical equations that can be used to investigate the tilt and the spherical contribution to the wavefront distortion induced to the laser beam by the effects of thermally induced natural convection next to the surface of thin-disk laser crystals. It was found that the angular tilt strongly increases with decreasing pump spot diameters. The dioptric power of the spherical contribution also increases with smaller pump spot sizes. These findings are considered very useful for the design of high-power thin-disk based laser architectures.

Acknowledgements Open Access funding provided by Projekt DEAL.

Funding European Union Seventh Framework Programme (ICT2013.3.2- Photonics, 619177).

Open Access This article is licensed under a Creative Commons Attribution 4.0 International License, which permits use, sharing, adaptation, distribution and reproduction in any medium or format, as long as you give appropriate credit to the original author(s) and the source, provide a link to the Creative Commons licence, and indicate if changes were made. The images or other third party material in this article are included in the article's Creative Commons licence, unless indicated otherwise in a credit line to the material. If material is not included in the article's Creative Commons licence and your intended use is not permitted by statutory regulation or exceeds the permitted use, you will need to obtain permission directly from the copyright holder. To view a copy of this licence, visit http://creativecommons.org/licenses/by/4.0/.

\section{References}

1. S. Chenais, F. Balembois, F. Druon, G. Lucas-Leclin, P. Georges, IEEE J. Quant. Electron. 40, 1217 (2004)

2. S. Chenais, F. Balembois, F. Druon, G. Lucas-Leclin, P. Georges, IEEE J. Quant. Electron. 40, 1235 (2004)

3. A. Giesen, H. Hügel, A. Voss, K. Wittig, U. Brauch, H. Opower, Appl. Phys. B 58, 365 (1994)

4. T. Dietrich, S. Piehler, C. Röcker, M. Rumpel, M.A. Ahmed, T. Graf, Opt. Lett. 42, 3263 (2017)

5. A. Diebold, F. Saltarelli, I.J. Graumann, C.J. Saraceno, C.R. Phillips, U. Keller, Opt. Express 26, 12648 (2018)

6. J.-P. Negel, A. Loescher, A. Voss, D. Bauer, D. Sutter, A. Killi, M.A. Ahmed, T. Graf, Opt. Express 23, 21064 (2015) 
7. T. Nubbemeyer, M. Kaumanns, M. Ueffing, M. Gorjan, A. Alismail, H. Fattahi, J. Brons, O. Pronin, H.G. Barros, Z. Major, T. Metzger, D. Sutter, F. Krausz, Opt. Lett. 42, 1381 (2017)

8. K. Schuhmann, K. Kirch, F. Nez, R. Pohl, A. Antognini, Appl. Opt. 55, $9022(2016)$

9. S. Piehler, T. Dietrich, P. Wittmüss, O. Sawodny, M.A. Ahmed, T. Graf, Opt. Express 25, 4254 (2017)

10. B. Weichelt, A. Voss, M.A. Ahmed, T. Graf, Opt. Lett. 37, 3045 (2012)

11. B. Weichelt, D. Blazquez-Sanchez, A. Austerschulte, A. Voss, T. Graf, A. Killi, 7721 (2010).

12. S.-S. Schad, V. Kuhn, T. Gottwald, V. Negoita, A. Killi, K. Wallmeroth, in edited by W. A. Clarkson and R. K. Shori (International Society for Optics and Photonics, 2014), vol. 8959, p. 89590U

13. Y.H. Peng, Y.X. Lim, J. Cheng, Y. Guo, Y.Y. Cheah, K.S. Lai, Opt. Lett. 38, 1709 (2013)
14. COMSOL Inc., "COMSOL Multiphysics", https://www.comso 1.com/. Accessed 18 Feb 2019.

15. K. Beil, S.T. Fredrich-Thornton, R. Peters, K. Petermann, G. Huber, in Optics InfoBase Conference Papers (Optical Society of America, 2009), p. WB28

16. Diamond Materials GmbH, The CVD diamond booklet. https:// www.diamond-materials.com/DE/cvd_diamond/overview.html. Accessed 18 Feb 2019.

17. J. A. Stone and J. H. Zimmerman, Index of refraction of air. https:// emtoolbox.nist.gov/Wavelength/Edlen.asp. Accessed 18 Feb 2019.

18. J.A. Stone, A. Stejskal, Metrologia 41, 189 (2004)

Publisher's Note Springer Nature remains neutral with regard to jurisdictional claims in published maps and institutional affiliations. 